\title{
LA DISTINCIÓN ENTRE LA ESFERA DE LO JURÍDICO A PRIORIY LA ESFERA MORAL
}

Mariano Crespo

RESUMEN: El propósito central de este trabajo es la reconstrucción sistemática y el anális is del "rendimiento" de la crítica que cierta orientación fenomeno ló gica del derecho, la eidética, realiza del iusnaturalismo. Para ello me voy a servir, fundamentalmente, de las contribuciones al respecto de Adolf Reinach y, en menor medida, de Dietrich von Hildebrand, en lo que se refiere a la distinción entre la esfera de lo jurídico a priori y la esfera de lo moral.

Palabras-llave: Reinach, von Hildebrand, a priori, iusnaturalismo, moral.

ABSTRACT: The aim of this paper is the systematic reconstruction and the analysis of the criticism that some phenomenological approaches do of classical naturalism. In order to show the difficulties of such approaches I refer to the distinction, made by Adolf Reinach and Dietrich von Hildebrand, between the a priori sphere of law and the moral sphere.

Keywords: Reinach, von Hildebrand, a priori, iusnaturalism, moral.

\section{Introducción}

El propósito central de este trabajo es la reconstrucción sistemática y el anális is del "rendimiento" de la crítica que cierta orientación fenomenoló gica del derecho, la eidética, realiza del iusnaturalismo. Para ello me voy a servir, fundamentalmente, de las contribuciones al respecto de Adolf Reinach ${ }^{1} \mathrm{y}$, en menor medida, de Dietrich von Hildebrand. ${ }^{2}$

\footnotetext{
1 (Reinach, 1989); (Reinach, 2010).

2 (Hildebrand, 1974).
} 
Expresado de esta forma tan sucinta podría dar la impresión de que de lo que aquí se trata es de una cuestión historiográfica sumamente particular $\mathrm{y}$, por tanto, de interés limitado. A mi juicio, dicha impresión estaría equivocada pues lo que está en juego en última instancia es una cuestión filosófica central, a saber, la peculiaridad de la esfera de lo legal o jurídico (rechtlich) frente a las esferas axiológica, moral y antropológica. Dicho de otro modo, la mencionada orientación eidética pone de manifiesto una serie de "entidades jurídicas" (rechtliche Gebilde) a priori, las cuales no tienen propiamente carácter normativo pero que, sin embargo, constituyen el fundamento de determinados derechos. Es el caso de la obligación y de la pretensión surgidas de una promesa o de la capacidad legal del propietario de transferir a otro su propiedad. Estos rechtliche Gebilde son diferentes tanto de los derechos naturales como de los derechos positivos, aunque están estrechamente relacionados con ellos. Es justamente en este contexto en el que se plantea la cuestión acerca de las semejanzas y diferencias entre este tipo de orientación fenomenológica al Derecho y el iusnaturalismo.

Ciertamente, el tipo de consideración fenomenológica del Derecho al que aquí me voy a referir, no es el único -tampoco el predominante- con el que ha sido considerado este fenómeno al interior del movimiento filosófico iniciado por Edmund Husserl. Junto con la orientación eidética, que considera al Derecho como una serie de conexiones esenciales, existen, como ha señalado recientemente (Loidolt, 2010, p. 46ss) al menos, tres direcciones u orientaciones fenomenológicas a esta cuestión: la dirección jurídica, la cual considera al Derecho como fenómeno del jurista; la dirección mundana, que estudia el Derecho como un fenómeno del mundo de la vida y la orientación trascendental, la cual se ocupa de las condiciones de posibilidad de la comprensión o constitución del Derecho. Esta última orientación es la que encontramos en la formulación madura del método fenomenológico que aparece en Ideas $I$.

En esa obra Husserl (Hua III/1, 354) muestra que la fenomenología tiene que recorrer el camino que va desde la constitución de la cosa material a la de todas las regiones de objetos. En este sentido, todo tipo peculiar de objeto - entre ellos, el Derechoexige su propia fenomenología constitutiva así como la consideración del entretejimie nto de las diferentes regiones. En este orden de cosas, las objetividades de orden superior que 
son las comunidades intersubjetivas, "aunque fundadas esencialmente en realidades psíquicas, que por su lado están fundadas en fisicas" (Husserl, ibid.), no pueden ser descritas en términos psicologistas y naturalistas.

\footnotetext{
“Así, todas las especies de objetos de valor y de objetos prácticos, todas las formaciones concretas de la cultura que determinan nuestra vida actual cual duras realidades, como, por ejemplo, el Estado, el Derecho, la costumbre, la iglesia, etc. Todas estas objetividades tienen que ser descritas tal como se dan, según sus especies fundamentales y en sus ordenaciones jerárquicas, planteando y resolviendo por respecto a ellas los problemas de la constitución" (Husserl, ibid.).
}

En esta orientación el Derecho es, considerado, por tanto como una formación de nuestra cultura, la cual ha llegado a constituirse y a sedimentarse de una determinada manera.

La orientación fenomenológico-eidética al Derecho surge, por el contrario, en el ámbito de la fenomenología temprana y de los primeros discípulos de Husserl (círculos de München y de Göttingen) y está en estrecha relación con el modo como este autor consideraba a la fenomenología en esa época inicial de su trabajo filosófico. ¿Cuáles son los elementos característicos fundamentales de esta orientación?

II. Elementos fundamentales de la orientación eidética al Derecho. La teoría a priori del Derecho de Adolf Reinach

Como es sabido, en la fenomenología temprana de Husserl se considera que el fin principal de ésta es el establecimiento de leyes eidéticas. El término "eidética" deriva del término "eidos" que Husserl introduce en Ideas I como equivalente de "esencia pura". "Eidética" significa lo mismo que "ciencia eidética". Como (Sowa, 2012, p. 254) recuerda, Husserl considera que la eidética es una ciencia a priori o no empírica en el sentido en el que "en sus proposiciones no se pone ni se presupone como existentes el mundo espacio-temporal o cualquier cosa real". Esto se aplica principalmente a dos 
disciplinas que nos son familiares, a saber, la aritmética pura y la geometría. Para Husserl éstas no sólo son dos modelos de ciencias eidéticas, sino que constituyen dos ejemplos de dos tipos fundamentales de éstas, a saber, las eidéticas formales y las eidéticas materiales. Esta distinción se basa, a su vez, en la existente entre el a priori formal (analítico) y el a priori material (sintético). Junto con la aritmética, dos son las ciencias que pertenecen a las "eidéticas formales". Me refiero a la lógica formal, orientada a las significaciones lingüísticas (conceptos, juicios, conclusiones, etc.) y que abstrae de todo contenido material de los conceptos y proposiciones particulares, y a la ontología formal, dedicada a las objetividades (estados de cosas, propiedades, etc.), la cual abstrae de todo contenido material perteneciente a una clase particular de objetos. Por su parte, las eidéticas materiales están vinculadas a dominios particulares de objetos (entidades físicas, entidades psíquicas, etc.). A ellas pertenecen no sólo la geometría y el resto de las llamadas ontologías regionales, sino también un tipo especial de eidética material, la eidética fenomenológica, entendida ésta como una "ciencia descriptiva no empírica de la esfera subjetiva e intersubjetiva de la constitución del mundo hecha accesible por medio de la reducción fenomenológica" (Sowa, 2012, p. 255).

En este contexto surge lo que podríamos denominar eidética del Derecho, entendiendo por tal una ontología regional en un doble sentido: por un lado, en el sentido del estudio del peculiar tipo de vivencias de la conciencia que tienen lugar en la esfera jurídica (por ejemplo, el prometer, el delegar, etc.) y, por otro lado, en el sentido del estudio de las objetividades que surgen de estas vivencias (como, por ejemplo, la pretensión y la obligación que nacen de un acto de prometer). Al Derecho, al igual que a cada ámbito de objetos, le corresponde una esfera de contenido apriórico, una legislación esencial a priori cuya investigación no es de tipo empírico. ${ }^{3}$

\footnotetext{
3 "Jedem gegenständlichen Gebiet ist eine Sphäre von apriorischem Gehalt, eine A priori Wesensgesetzlichkeit zugeordnet, und diese Sphäre ist vor aller empirischen Feststellung zu untersuchen... Für jede empirische Wissenschaft gibt es eine rationale, die Wesenszusammenhänge, apriorische Notwendigkeiten, Möglichkeiten, Unverträglichkeiten u.dgl. aufstellt" (Reinach, 1989, p. 440); "Es fehlt jedes, aber auch jedes Recht dazu, das Apriori auf das Formale in irgendeinem Sinne zu beschränken, auch von dem Materialen, ja dem Sinnlichen, von Tönen und Farben gelten apriorische Gesetze. Damit öffnet sich der Forschung
} 
Es justamente a la exploración de esta esfera a lo que Adolf Reinach dedicó su obra más importante, a saber, Los fundamentos a priori del Derecho civil. Reinach, el cual conocía con profundidad el pensamiento de Husserl -fue su asistente durante el tiempo en el que éste fue profesor en la Universidad de Göttingen-, se dio pronto cuenta que hay toda una clase de objetos que son temporales, pero que por ello no son ni psíquicos ni físicos. En este orden de cosas, uno de los objetivos de sus Fundamentos es, justamente, poner de manifiesto la peculiaridad ontológica de estas entidades cuyo ser es independiente de su captación por los sujetos conscientes. En esta obra Reinach pone de relieve el peculiar estatuto ontológico de objetividades como pretensiones y obligaciones surgidas de actos sociales como las promesas. Éstas surgen, duran y, normalmente, se extinguen. Así cuando un hombre promete algo a otro, ello provoca, si cabe hablar así, un cierto efecto en la persona destinataria de la promesa. Una vez efectuada la promesa y, por tanto, una vez surgidas la pretensión y la obligación correspondientes, pueden suceder dos cosas: o bien que la promesa se cumpla y, por tanto, deje de existir como tal o bien que la persona destinataria de la misma renuncie a su cumplimiento, con lo cual también podemos decir que la promesa deja de existir.

Una de las características de estas objetividades - de las que las pretensiones y las obligaciones que surgen de una promesa constituyen un ejemplo- puede observarse a través del examen de los estados de cosas que en ellas se fundan. Que una pretensión se extinga por un acto de renuncia se basa en la esencia de la pretensión como tal. No se trata aquí de "forzosidad psíquica" alguna, de un tener-que-pensar-así-y-no-poder-pensar-deotro-modo, sino de un-ser-así-y-no-poder-ser-de-otro-modo. Esta necesidad no lo es, por tanto, de la esfera de la aprehensión de los estados de cosas sino de los estados de cosas mismos. Al mismo tiempo, estas conexiones esenciales no valen sólo para, en nuestro ejemplo, esta pretensión particular, sino que convienen absoluta y necesariamente a todo lo que es de tal especie.

ein Gebiet, so groß und reich, daß wir es heute noch gar nicht übersehen können" (Reinach, 1989, pp. 546-547). 
De este modo, Reinach funda una teoría a priori del Derecho, la cual se ocuparía de schlichte Seinsgesetze referidas a entidades jurídicas independientes de cualquier ley positiva. Se trata, pues, de una "eidética material" que estudia la legalidad esencial existente en de la esfera de lo rechtlich. Una de estas leyes es, por ejemplo, la que afirma que una promesa se extingue cuando se cumple la misma o cuando el destinatario de ella renuncia a su pretensión. Reinach sostiene que los conceptos básicos del Derecho tienen un ser que es independiente de la ley positiva, del mismo modo que los números tienen un ser independiente de la ciencia matemática. La ley positiva puede desarrollarlos y transformarlos, pero ellos mismos no son producidos por ella, sino tan sólo encontrados. Las leyes que gobiernan estas entidades y estructuras son independientes de nuestra comprensión de las mismas como lo son las leyes de las matemáticas.

\section{Teoría a priori del Derecho e iusnaturalismo}

Por lo que acabo de explicar en este apretado resumen de la eidética material o teoría a priori del Derecho propuesta por Reinach, resulta claro que ésta constituye una crítica a la consideración de que las únicas normas que existen son las que, directa o indirectamente, se derivan de la ley [positiva] y a la consideración de que las normas son exclusivamente productos de la voluntad del legislador. Supone, por tanto, la defensa de una serie de elementos pre-positivos, los cuales tienen un cierto efecto regulador sobre las leyes positivas. ${ }^{4}$ Entre estos elementos destaca un grupo que es el que a nosotros nos interesa analizar en este contexto. Me refiero a lo que, en términos generales, podemos denominar la "naturaleza de las cosas". Sin ánimo de ser exhaustivo, permítanme que me refiera a los diversos sentidos de esta expresión. Mi objetivo es clarificar si el uso que de

\footnotetext{
${ }^{4}$ En varios de sus escritos W. Waldstein se ha referido a estos elementos prepositivos en el Derecho romano, en particular, y en el Derecho, en general. Éstos son: las características o propiedades de las cosas que regular; lo que los romanos llamaron fides, en el sentido de autenticidad, honradez, confianza, en contraposición a malicia, engaño, etc.; la aequitas, iustitia, iustum y semejantes; el ius naturale y, por último, la estructura inteligible de las cosas. Cf. (Waldstein, 1967); (Waldstein, 2010).
} 
la misma hacen el iusnaturalismo clásico y la teoría a priori del Derecho -cuando ésta se refiere a la naturaleza inteligible de las cosas- es el mismo.

a) Un primer sentido que la expresión "naturaleza de las cosas" puede tener es, justamente, el que está presente en la tradición del iusnaturalismo clásico, el cual está ligado a una metafísica de la creación. Lo importante para nosotros aquí es la idea de que la naturaleza o esencia de una cosa es el fundamento por el cual se dice que esta cosa es lo que es, su tipo de ser, lo que hace que ella sea lo que es. ${ }^{5}$

b) Un segundo sentido que el término "naturaleza" puede adquirir es el referido a la naturaleza fáctica de las cosas en el sentido de las características o propiedades de los objetos que regular. El que las cosas sean de un determinado modo y no de otro es el origen de ciertas prescripciones. Así, por ejemplo, la "naturaleza" del tráfico marítimo, de la relación entre padres e hijos, de los objetos indivisibles, etc. tiene consecuencias prescriptivas. Esto explicaría, por ejemplo, el absurdo de tratar a los animales vivos del mismo modo que se trata a los objetos que son divisibles en partes. Tampoco tendría sentido tratar a los bienes inmuebles del mismo modo que tratamos a los animales. ${ }^{6}$

c) Un tercer sentido de "naturaleza de las cosas" es el presente en la "teoría de la naturaleza de las cosas" defendida entre otros por autores como Gustav Radbruch. ${ }^{7}$ Ciertamente, detrás de esta teoría se encuentra una concepción de la naturaleza diferente de la que sostienen los iusnaturalistas clásicos. Radbruch crítica precisamente esta concepción en la medida en que, a partir de la naturaleza del hombre, pretende fundar el mismo Derecho para todas las épocas y para todos los pueblos. Él considera, por el contrario, que de la naturaleza de las cosas se deriva una multiplicidad de formaciones

\footnotetext{
${ }^{5}$ Cf., por ejemplo, SummaTheologiae, I-II, q. 90ss. Un buen resumen se encuentra en (MayerMaly, 1998).

6 Cf., por ejemplo, (Gutzwiller, 1965) y (Schambeck H., 1965, p. 172ss).

7 Cf., por ejemplo, (Radbruch, 1990).
} 
jurídicas históricas y nacionales. ${ }^{8}$ En esta teoría la naturaleza o esencia de las cosas es vista, más bien, como el sentido objetivo de éstas que surge de las características de las Lebensverhältnisse, “como la respuesta a la pregunta: ¿cómo puede esta Lebensverhältnis así constituida ser pensada con sentido, esto es, como realización de una idea y de qué idea?". 9 La naturaleza de la cosa es el sentido de una Lebensverhältnis fáctica. ${ }^{10}$

d) Un cuarto sentido de la expresión "naturaleza de las cosas" es el presente en la concepción funcionalista de Norberto Bobbio. Como es sabido para este autor, la naturaleza de un objeto o de un sujeto o de un comportamiento o de una institución hace alusión a la relación medio - fin, esto es a la idoneidad de estas entidades para "funcionar como medio para conseguir ciertos fines". ${ }^{11}$ La naturaleza de las cosas es, pues, "el medio que se considera más apto para alcanzar un cierto fin". ${ }^{12}$ Esto lleva a este autor a proponer, incluso, la sustitución de la expresión "naturaleza de las cosas" por la de "función económico-social" de una institución jurídica. ${ }^{13}$

Ciertamente, como decía hace un momento, esta lista de posibles sentidos de la expresión "naturaleza de las cosas" no es exhaustiva. En cualquier caso, pareciera que los apriorische rechtliche Gebilde, en cuanto estructuras inteligibles, que constituyen los fundamentos de la eidética fenomenológica del Derecho o teoría a priori del Derecho

8 "Man darf die Natur der Sache nicht als eine naturrechtliche Denkform missverstehen. Natur der Sache und Naturrecht sind viel eher Gegensätze. Das Naturrecht, aus der Natur des Menschen: der Vernunft hergeleitet, beansprucht ein für alle Zeiten und Völker gleiches Recht zu begründen, aus der Natur der Sache aber ergibt sich die Mannigfaltigkeit historischer und nationaler Rechtsbildungen. Sie ist deshalb eminent geeignet, historischem, nationalem, konservativem Rechtsdenken zur Grundlage zu dienen" (Radbruch, 1990, p. 230).

9 "Natur der Sache ist ihr Wesen, ihr Sinn, und zwar nicht ein von irgendjemandem wirklich gedachter, vielmehr der allein aus der Beschaffenheit der Lebensverhältnisse selbst zu entnehmende objektive Sinn, die Antwort auf die Frage: wie kann dieses so beschaffene Lebensverhältnisse als sinnvoll gedacht werden, d.h. als Verwirklichung einer Idee - und welcher Idee? Und zwar wird gesucht der juristische Sinn und die Rechtsidee, die sich in ihm verwirklicht" (Radbruch, 1990, pp. 234-235).

${ }^{10}$ Cf. (Radbruch, 1990, p. 236).

11 Cf. (Bobbio, 1958).

12 Ibid., p. 204.

13 Ibid., p. 212. 
propuesta por Adolf Reinach y, en cierta medida, por Dietrich von Hildebrand, están más cerca del primer sentido de naturaleza de las cosas al que me he referido, a saber, el defendido por el iusnaturalismo clásico, que de los otros sentidos de esta expresión. Como he tenido ocasión de comentar más arriba, Reinach sostiene que estos rechtliche Gebilde existen con independencia del Derecho positivo, el cual los presupone y usa ${ }^{14}$ y aparecen en éste cuando se habla de la "naturaleza de las cosas":

\footnotetext{
"Las entidades jurídicas existen independientemente del Derecho positivo, pero son presupuestas y utilizadas por él. Por esta razón, su análisis y la clarificación de su esencia (clarificación inmanente e intuitiva) puede resultar importante para las disciplinas del Derecho positivo. También las leyes que se fundan en su esencia desempeñan en el Derecho positivo un papel mucho mayor del que pudiera sospecharse. Es sabido cuán frecuentemente se habla en la jurisprudencia de proposiciones que, sin ser de derecho escrito, "son autoevidentes» $\mathrm{o}$ «resultan de la naturaleza de la cosa», por mencionar tan sólo algunas de estas expresiones. En la mayoría de los casos no se trata, como se ha creído, de proposiciones cuya utilidad práctica o cuya justicia son evidentes sin más, sino más bien de leyes de la teoría a priori del Derecho. En realidad, se trata de proposiciones que resultan de la «naturaleza» o de la «esencia» de los conceptos en cuestión”. ${ }^{15}$
}

Sin embargo y a pesar de reconocer al iusnaturalismo el "mérito" de haber reconocido que las conexiones jurídicas existen con independencia de las determinaciones positivas, Reinach dedica las páginas finales de sus Fundamentos a priori del Derecho civil a esforzarse por mostrar las diferencias entre la teoría del Derecho a priori y el iusnaturalismo. Reinach ve las principales diferencias entre ambas teorías sobre todo en dos puntos en los cuales el iusnaturalismo habría fallado: por un lado, en el proyecto de un Derecho material que tenga también validez general incondicionada $\mathrm{y}$, por

\footnotetext{
14 (Reinach, 1989, p. 146).

15 (Reinach, 2010, p. 26); "Wenn Phänomenologie die 'auf generelle Essenzen gerichtete, rein aus dem Wesen rein ihre Geltung schöpfende Erkenntnis' ist (Die Idee der Phänomenologie, 51), so sind juristische Denkformen wie die 'Natur der Sache', die dem Recht vorgegebenen 'sachlogischen Strukturen', Betrachtungen über das 'Wesen' des Eigentums oder der Verpflichtung in dieser Bestimmung des Gegenstandes phänomenologischen Denkens ohne weiteres einbegriffen" (Bodenheimer, 1969, p. 89).
} 
otro, en el modo de considerar el Derecho positivo. Por lo que se refiere a lo primero, Reinach reprocha al iusnaturalismo el haber creído en "la posibilidad de establecer para siempre un Derecho ideal de contenido inmutable sin prestar atención a la mutabilidad de las condiciones de vida de las cuales depende la validez de tales proposiciones". ${ }^{16}$ Por su parte, Reinach insiste en que su teoría a priori del Derecho no opera en un plano normativo, no pretende ofrecer Derecho superior alguno, sino tan sólo "simples leyes del ser" (schlichte Seinsgesetze), según las cuales surgen las relaciones jurídicas como, por ejemplo, las obligaciones y pretensiones de un acto de prometer. ${ }^{17}$ Se trata, por tanto, de proposiciones teoréticas -no normativas- fundadas en la esencia de ciertos actos y situaciones. ${ }^{18}$ Por tanto, a Reinach no le interesa el contenido de las pretensiones y obligaciones, sino su lógica misma. ${ }^{19}$ Volveré más adelante sobre la relevancia de este punto en el planteamiento de este autor.

Por lo que se refiere a la segunda diferencia entre su teoría del Derecho $a$ priori y el iusnaturalismo, a saber, el modo de considerar el Derecho positivo, Reinach no entra directamente a explicar dicha diferencia, sino que se limita a explicar su propia concepción de las capacidades del Derecho positivo dejando entrever una crítica al

16 (Reinach, 2010, p. 157).

17 "Der gedankliche Rückgriff auf den 'Sinneskern' von Rechtssachverhalten, denen wir in der Rechtswirklichkeit begegnen, eröffnet uns (wenn fehlerfrei durchgeführt) die Sicht auf ein System, nicht von Verhaltensnormen 'höherer Stufe', sondern von Rechtswahrheiten, die als solche keine normative Kraft besitzen. Eine Reduktion, die das leistet, enthüllt uns die Grundstrukturen jedes möglichen Rechts; sie haben die Natur eines juristischen Apriori" (Husserl, 1955, p. 14).

18 "Es handelt sich ja hierbei nicht um apriorische Normen, die dem positiven Recht gegenüber gestellt werden können, wie das a priori sittlich gefordert Verhalten der Menschen dem tatsächlichen Handeln der Menschen, das jenem häufig widerspricht, indem es unsittlich ist. Vielmehr betrifft dieses apriorische Recht durchaus nicht bloße Normen - ein sein-Sollendes sondern es sind die, unabhängig von allem positiven Recht, in dem Wesen gewisser Akte und Situationen an sich fundierten rechtlichen Gebilde, die, sofern jede positive rechtliche, diesbezügliche Bestimmung fehlt, ohne weiteres bestehen und objektiv 'rechtens' sind" (Hildebrand, 1974, p. 405).

19 "Si le droit naturel représente, comme c'est le cas bien souvent, la part de la morale dans le droit, il est clair que Reinach n'a rien à voir avec la tradition iusnaturaliste. Son effort a tendu seulement à montrer que les lois sont, pour une bonne part d'entre elles, selon la formule de Montesquieu 'les rapports nécessaires qui dérivent de la nature des choses', pourvu que par nature des choses on n'entende pas la nature physico-chimique à laquelle Montesquieu ne se privai pas de faire appel, mais l'essence même des institutions" (Gardies, 1972, p. 185). 
iusnaturalismo. Pareciera que éste no habría tomado suficientemente en serio el "poder creador" del mismo. Hildebrand, el cual desarrolla la teoría de Reinach, sostiene:

\begin{abstract}
"Asícomo el prometer, el ordenar, el regalar y otros actos sociales poseen es encialmente esta fuerza creadora de traer al mundo entidades jurídicas, así también el peculiar acto de determinación (Bestimmungsakt) de la instancia jurídica autoritativa posee, en virtud de su es encia, una potencia peculiar en sentido jurídico". ${ }^{20}$
\end{abstract}

Así, por ejemplo, la promulgación, por parte de la instancia legisladora competente, por ejemplo, el Estado, de que los ciudadanos adquieran la mayoría de edad a los 18 años, "no es el reconocimiento de un derecho o de un precepto existente con anterioridad, sino la creación real de una entidad jurídica real". ${ }^{21}$ Esto supone, como con claridad señala Hildebrand, distinguir entre dos casos: por un lado, entidades jurídicas que no existirían si no hubiera Derecho positivo, esto es, relaciones jurídicas que no pueden desviarse de condiciones a priori de constitución de tales entidades sencillamente porque no existen tales condiciones. Se trata únicamente de "creaciones" de instancias legislativas como, por ejemplo, la mayoría de edad o la capacidad de participar en elecciones o que una ley exija la aprobación de las dos cámaras parlamentarias, etc. Por otro lado, nos encontramos con los casos en los que determinadas promulgaciones o disposiciones positivas por parte de la instancia legislativa competente se apartan de las condiciones a priori de constitución de ciertas entidades jurídicas. Es lo que sucede cuando, por ejemplo, una ley determina que una obligación surge de una promesa sólo cuando ésta se realiza por escrito o que un acto de regalar o transferir una propiedad sólo es válido cuando se realiza ante notario. ${ }^{22}$

Esta posibilidad de que leyes positivas se desvíen de leyes a priori es explicada, tanto por Reinach como por Hildebrand, recurriendo a los dos tipos diferentes de proposiciones y de actos subyacentes a éstas que se dan en el Derecho a priori y en el

\footnotetext{
20 (Hildebrand, 1974, p. 406).

21 Ibid.

22 Las explicaciones de Hildebrand al respecto son, a mi juicio, mucho más esclarecedoras que las del propio Reinach. Cf. (Hildebrand, 1974, pp. 406-407).
} 
Derecho positivo. Mientras que en el caso de las leyes de Derecho positivo se trata, como vimos en el caso de Reinach, de simples leyes del ser a las cuales subyacen Anpassungsakte, en el caso de las leyes positivas se trata de proposiciones normativas en cuya base se encuentran Bestimmungsakte. Si las proposiciones de las leyes positivas fueran meros juicios que constatan un estado de cosas, entonces entrarían en contradicción con las proposiciones del Derecho a priori y una de las dos debería ser falsa. Sin embargo, en el Bestimmungsakt no se trata de un Behaupten, sino de un Schaffen de algo nuevo. No puede, por tanto, haber contradicción alguna entre proposiciones, en última instancia, descriptivas y proposiciones prescriptivas. $^{23}$

En estrecha relación con lo que acabamos de señalar, Reinach expone sus reservas con respecto a la expresión "Derecho natural". Éstas giran en torno a la inadecuación de la misma para designar la tendencia -valorada positivamente por él- a la búsqueda de elementos pre-positivos, "pues no se trata aquí ni de un «Derecho» ni la «naturaleza» desempeña un papel constitutivo". ${ }^{24}$ Según Reinach, el iusnaturalismo comete un triple error por lo que se refiere a la "naturaleza". En primer lugar, "se pensó que la validez general de las leyes jurídicas procede del hecho de que la «naturaleza» es la misma en todos los hombres que la descubren". ${ }^{25}$ Sin embargo, las relaciones jurídicas no derivan su objetividad del hecho de que estén inscritas en la naturaleza del ser humano. Éstas son, como Reinach insiste, independiente de la constitución fáctica del ser humano y de comportamiento. Del mismo modo, sería falso considerar estas leyes como basadas en algo así como el consentimiento universal de la misma forma que lo sería concebir las leyes de la aritmética como fundadas en un supuesto acuerdo universal. El hecho de que seamos nosotros, con nuestra peculiar constitución mental, los que intuyamos el que una

23 "Nie und nimmer kann eine positive Rechtsinstanz dem Versprechen an sich seine rechtlich schöpferische Kraft rauben, ebensowenig wie sie inm diese erst verleihen kann. Sie besteht, ob die positive Rechtsinstanz sie anerkennt oder nicht. . Wohl aber kann die positive Rechtsinstanz durch einen ebenfalls wesenhaft rechtlich schöpferischen Akt, der eben keine Anerkennung oder ein Urteil irgendwelcher Art, sondern ein Bestimmungsakt ist, erreichen, das sin ihrem Kompetenzbereich das Versprechen nur unter gewissen Bedingungen seine rechtliche Wirksamkeit entfaltet" (Hildebrand, 1974, p. 407).

24 (Reinach, 2010, p. 158).

25 Ibid., p. 159. 
promesa se extinga por un acto de renuncia, no significa que lo intuido deba a nosotros su validez. El segundo error cometido por los defensores de la existencia del Derecho natural habría sido -siempre según Reinach- pensar que las leyes del mismo "valen sólo para el hombre o, a lo sumo, para seres de la misma o semejante 'naturaleza" "26 cuando en realidad las leyes referidas a entidades jurídicas se basan en la esencia de ellas mismas y en las de los actos de los que surgen. Por eso, éstas son válidas no sólo para este mundo, sino para cualquier mundo posible. En tercer y último lugar, se buscaban estas leyes a priori en la esfera de la naturaleza, esto es de lo fisico y de lo psíquico. Sin embargo, como es sabido, Reinach insiste en que las relaciones jurídicas que surgen de los actos sociales no son ni de carácter físico ni de carácter psíquico.

En resumen, nos encontramos frente a objetividades del todo peculiares y puramente jurídicas. $\mathrm{Y}$ así las leyes esenciales que valen para ellas no deberían en modo alguno ser consideradas como leyes de la naturaleza. De este modo, Reinach inaugura un “dominio de leyes puramente jurídicas que subsisten independientemente de la «naturaleza», independientemente del conocimiento y de la organización humanos $\mathrm{y}$, sobre todo, independientemente de la evolución real del mundo". ${ }^{27}$

IV. El principal rendimiento de la crítica de Reinach al iusnaturalismo: la especificidad de la esfera de lo rechtlich a priori frente a la esfera moral

Como mencionaba hace un momento, la crítica que Adolf Reinach realiza del iusnaturalismo en Los fundamentos a priori del Derecho civil se condensa en siete apretadas páginas. El hecho de que este autor muriera durante la Primera Guerra Mundial, cuatro años después de la publicación de esta obra, supuso la trágica interrupción de una prometedora carrera filosófica en la que seguramente muchos de los puntos tan sólo esbozados en ese libro hubieran encontrado su desarrollo. Seguramente hubiera tenido la

\footnotetext{
26 Ibid.

27 Ibid., p. 161.
} 
oportunidad de profundizar en su comprensión del iusnaturalismo clásico y en la noción de "naturaleza" que éste maneja. Ello le hubiera llevado, a mi juicio, a reconsiderar su crítica a esta posición y a reconsiderar la cuestión de las semejanzas y diferencias entre su teoría a priori del Derecho y la tradición del Derecho natural.

En esta segunda parte de mi contribución no quisiera realizar una "crítica de la crítica" que Reinach lleva acabo del iusnaturalismo. Tan sólo quisiera plantear lo que podríamos denominar el "rendimiento" filosófico de la crítica de Reinach al iusnaturalismo, a saber, el haber mostrado la especificidad de la esfera de lo jurídico (rechtlich) frente a otras esferas como la moral, la axiológica y la antropológica. Salvo Dietrich von Hildebrand en un artículo poco conocido, ${ }^{28}$ no han sido muchos los estudiosos del pensamiento de Reinach que han analizado este aspecto. ${ }^{29}$ La clarificación de la diferencia entre ambas esferas constituye, a su vez, un primer paso para comprender cabalmente la distinción entre teoría a priori del Derecho y el iusnaturalismo.

Una distinción preliminar realizada por Hildebrand y que tiene que ver con las cuestiones señaladas por Reinach en Los fundamentos a priori... es la distinción entre dos sentidos de Recht y, por consiguiente, de rechtliche Gebilde. Con rechtliche Gebilde en sentido estricto se alude a las vinculaciones legales que surgen de determinados actos. Un ejemplo sería la capacidad legal del propietario de una cosa de regalarla a otro sujeto. A este ámbito pertenecen las entidades jurídicas así como las leyes fundadas en ellas que, según Reinach, constituyen los fundamentos a priori del Derecho. Recuérdese que estos fundamentos no tienen un carácter normativo y que tan sólo reflejan schlichte Seinsgesetze. Al segundo sentido de Recht -lo que Hildebrand denomina Rechte in weiteren Sinn- se refiere, en primera línea, el Derecho natural. Un ejemplo de un derecho en este sentido es el que posee todo ser humano a decidir libremente en cuestiones de conciencia. A diferencia de los derechos en sentido estricto, los derechos en este sentido tienen un carácter normativo.

\footnotetext{
28 (Hildebrand, 1974).

29 Una excepción son (Albert, 2013a) y (Albert, 2013b).
} 
La esfera de lo rechtlich y la esfera de lo sittlich son dos mundos diferentes, aunque relacionados. Es muy importante reconocer la peculiaridad de cada una de estas esferas y no pretender que una sustituya a la otra. Un gran número de errores tiene precisamente su origen en la confusión de ambas esferas. Se trata de dominios del ser completamente diferentes. Lo que tenemos in mente cuando hablamos de la generosidad de un acto de perdón es muy diferente de lo que pensamos cuando hablamos del derecho de una persona a disponer de su propiedad o de las rechtliche Verbindlichkeiten que surgen de un contrato.

La diferencia entre la esfera de lo rechtlich y la de lo sittlich queda también de manifiesto si comparamos la rechtliche Verbindlichkeit que surge, por ejemplo, del prometer algo a alguien con la moralische Verpflichtung de cumplir esa promesa. La primera presupone siempre un Partner frente a la que existe. Sólo ante aquel al que he prometido algo existe la Verbindlichkeit de cumplir lo prometido y no frente a una tercera persona. Como Reinach pone de manifiesto, el destinatario de mi promesa puede suprimir esta Verbindlichkeit mediante un acto de renuncia. Sin embargo, la obligación moral de cumplir lo prometido no la tengo frente al destinatario de la promesa, sino que la tengo en sí misma. ${ }^{30}$ Por esa misma razón, él no me puede dispensar de ella. Sólo un cambio de la situación puede suprimir la obligación. ${ }^{31}$ En definitiva, todas las vinculaciones morales se fundan en valores, pero las legales no necesariamente.

\footnotetext{
30 (Hildebrand, 1974, p. 404).

31 "Von moralischen Verpflichtungen, die ich jemand gegenüber habe, kann mich der Betreffende so wenig dispensieren wie ein dritter. Nur eine Veränderung der Sachlage kann die Verpflichtung aufheben. Auch von der moralischen Verpflichtung, das Versprochene zu halten, kann mich der andere, dem ich es versprach, nicht direkt dispensieren; er kann nur durch einen Verzicht die Verbindlichkeit vernichten und damit indirekt die objektive Sachlage so verändern, dass die moralische Verpflichtung auch erlischt. Die 'Relativiät' der rechtlichen Verbindlichkeit auf einen Partner trennt diese deutlich von der stets partnerlosen sittlichen Verpflichtung" (Hildebrand, 1974, p. 404).
} 
V. Derechos a priori, derechos naturales y Derechos positivos

Como decía hace un momento, la clarificación de la diferencia entre la esfera de lo rechtlich y la esfera de lo sittlich constituye, a su vez, un primer paso para comprender cabalmente la distinción entre teoría a priori del Derecho e iusnaturalismo. Los siguientes pasos orientados a la comprensión de esta distinción son, según Hildebrand, los siguientes:

a) En primer lugar, es preciso comprender la peculiaridad de los derechos naturales frente a los derechos a priori, a los derechos positivos y a los derechos morales.

b) En segundo lugar, es necesario esclarecer la relación entre Derecho a priori y Derecho positivo.

c) En tercer lugar, hay que explicar la relación entre Derechos positivos y Derechos morales

Por lo que se refiere a la primera de las tareas, a saber, el esclarecimiento de la peculiaridad de los derechos naturales frente al resto de los derechos, Hildebrand parte con un ejemplo, a saber, la consideración del Derecho natural del ser humano al libre desarrollo de sus capacidades espirituales. Resulta claro que en este caso hablamos de "derecho" en un sentido diferente a cuando hablamos de los derechos que se adquieren cuando se alcanza la mayoría de edad. En este último caso, cuando el sujeto alcanza una edad determinada, por ejemplo, los 18 años, adquiere una serie de capacidades o potencialidades de las cuales antes no disponía (por ejemplo, el derecho a participar en las elecciones como elector y como candidato). Por su parte, el derecho a desarrollar libremente sus capacidades espirituales es un derecho que todo ser humano tiene por el simple hecho de serlo, por su naturaleza como persona. Si una legislación positiva estableciera un derecho como el antiguo Züchtigungsrecht existiría un derecho positivo tal en cuanto realidad legal (rechtliche Realität). Sin embargo, no existe un derecho natural a la Züchtigung. Es más, nos parecería que la supresión de ese derecho positivo 
constituiría una equiparación de la situación legal con las exigencias del Derecho natural y de los derechos "morales" de la mujer y de los hijos. Por consiguiente, de lo que se trata, según Hildebrand, es de comprender que los derechos naturales son algo propio, diferentes, por un lado, de los derechos a priori y de los derechos positivos, y por otro, a pesar de su "pathos" ético, de lo moralmente valioso y de lo moralmente obligatorio. ${ }^{32}$ También es importante diferenciar el Derecho natural de la categoría de lo moralmente permitido. Cuando decimos que el ser humano, en cuanto persona espiritual, tiene derecho a la libertad de conciencia, decimos mucho más que simplemente que le está permitido actuar según su conciencia.

Ciertamente, me está moralmente permitido hacer uso de ese derecho, aunque tampoco es moralmente obligatorio hacer uso de todo derecho moral. Es tan sólo moralmente permitido. En este punto, Hildebrand esboza un interesante análisis de la categoría de "permisibilidad" (Erlaubtheit) en el que pone de relieve que ésta es una delimitación puramente negativa frente a lo prohibido, que coincide con "no prohibido", pero que, no obstante, no significa lo mismo que el derecho moral a algo. Un derecho natural es algo mucho más positivo y lleno de contenido que algo simplemente permitido. La diferencia entre un derecho natural y, por ejemplo, un determinado comportamie nto permitido se manifiesta -sostiene Hildebrand en un pasaje no especialmente claro- en el hecho de que sólo un comportamiento propio puede ser permitido. Por el contrario, el derecho natural se puede referir tanto a un comportamiento propio como a uno ajeno. Así, por ejemplo, el ser humano tiene un derecho moral a una cierta confianza por parte de los otros hombres o a la buena fama. También estos derechos morales son a priori, es decir, fundados en la esencia de la persona espiritual y de determinadas situaciones. Así, el derecho moral del ser humano a la libertad de conciencia se funda en la esencia de la persona espiritual, el derecho de los padres a la educación de los hijos en la esencia de la paternidad, etc. En estos derechos se trata siempre de algo que es válido a priori, con

32 "Es handelt sich nur für uns darum, einzusehen, dass diese Naturrechte etwas Eigenes darstellen, das einerseits nicht mit den Rechten in engeren Sinn, seien es apriorische oder positive, identifiziert werden darf und anderseits trotz seines immanente ethischen Pathos von dem sittlich Wertvollen und sittlich Verpflichtenden kategorial verschieden ist" (Hildebrand, 1974, p. 408). 
independencia de todo cambio de los tiempos, de las costumbres y de las ideas humanas. Sin embargo, no todos estos derechos están en el mismo nivel en lo que se refiere a su imperdibilidad (Unverlierbarkeit). Algunos de estos derechos pueden perderse o verse limitados en su ejercicio en virtud de un determinado comportamiento moralmente negativo (como, por ejemplo, el derecho a la libertad de movimiento cuando se comete un delito, el derecho de los padres a educar a los hijos cuando estos son maltratados o abusados, etc.). Todo lo que acabamos de señalar pondría de manifiesto, según Hildebrand, la peculiaridad de los derechos naturales frente a la esfera de los valores morales y de los vínculos morales, a pesar de las relaciones que existen entre estas dos esferas. $^{33}$

Los derechos naturales son también diferentes tanto de los derechos positivos como de los derechos a priori. Esta diferencia puede observarse, piensa Hildebrand, si comparamos la "fuerza jurídica creadora" (rechtliche schöpferische Kraft) que tiene el acto por medio del cual el propietario de una cosa transmite su propiedad a otra persona, el cual nace de un poder legal (rechtliches Können), con el derecho de todo hombre a la libertad de conciencia o a la libertad de expresión. Este derecho no concede ningún poder legal. Se trata tan sólo de una pretensión (Anspruch) objetivamente fundada con un "pathos" moral diferente de lo puramente legal y que constituye una norma para el Derecho positivo contra la cual éste no debe atentar. Por su parte, un derecho a priori, en el sentido en que Reinach y el propio Hildebrand utilizan esta expresión, no constituye una norma para el Derecho positivo. Es más, el Derecho positivo puede y, a veces, incluso debe en ciertas circunstancias y por razones morales desviarse de los derechos a priori (por ejemplo, anulando una promesa efectuada por un menor de edad o que atenta contra los derechos naturales). De lo que no puede desviarse es de los derechos naturales de la persona o de una comunidad. No puede anularlos ni impedirlos. Si lo hiciera, se

\footnotetext{
33 'So stellen diese 'Naturrechte' im engeren Sinn ein Eigenes gegenüber der Sphäre sittlicher Werte und sittlicher Bindungen dar. Ob man dieselben trotzdem als eine eigene Kategorie in die Sphäre des Sittlichen einbezieht wegen ihres sittlichen 'Ethos', ist für uns hier von untergeordneter Bedeutung. Es genügt, wenn ihre Eigentümlichkeit gegenüber der Sphäre der sittlichen Werte und Bindungen klar erkannt wird und ihre kategoriale Eigenart als echte 'Rechte"' (Hildebrand, 1974, p. 409).
} 
convertiría en un derecho injusto, aunque válido legalmente. En resumen, los derechos naturales están más allá de la esfera de los derechos positivos. Sólo la persona misma, en virtud de ciertos comportamientos negativos, puede perder ciertos derechos naturales.

Todo esto abunda en la separación entre Derechos naturales, derechos a priori y derechos positivos. En este orden de cosas, Hildebrand, en clara continuidad con los anális is iniciados por Reinach en Los fundamentos a priori del Derecho civil, se ha esforzado por mostrar que la esfera de lo rechtlich, en el sentido más amplio de la palabra, se diferencia como una categoría propia de la esfera de lo valioso en general, ya sean valores morales, estéticos o de otro tipo, a pesar de que la esfera de los derechos naturales nos ha acercado más a la esfera moral. Sin embargo, esta diferencia no significa que no existan relaciones entre ellas. Así, por ejemplo, lo moral ejerce un papel normativo con respecto al derecho positivo y al derecho a priori. ${ }^{34}$ En este sentido, si el ejercicio de un derecho a priori como, por ejemplo, la obligación de cumplir una promesa, lleva a la realización de una acción inmoral, el Derecho positivo debe introducir una modificación que ponga remedio a esa situación. Es lo que sucede en las limitaciones que la legislación de un país puede establecer a los contratos contra bonos mores. La vinculación legal, que surge de este contrato, es en principio independiente del contenido del mismo. Si éste es inmoral, surge entonces un conflicto entre la obligación (Verpflichtung) de considerar la vinculación legal (rechtliche Verbindlichkeit) y la obligación de no llevar a cabo una acción inmoral, un conflicto en el cual la consideración del contenido tiene preferencia. Por su parte, el derecho positivo puede eliminar definitivamente este conflicto mediante la promulgación de una disposición que elimine toda vinculación legal que se refiera a un contenido inmoral. No obstante, con ello, señala Hildebrand, no se soluciona definitivamente el conflicto puesto que lo que el Estado declara como inmoral o contra bonos mores puede no coincid ir totalmente con lo objetivamente prohibido moralmente o con la conciencia subjetiva de lo tenido por no permitido moralmente en el caso individual. ${ }^{35}$

\footnotetext{
34 (Hildebrand, 1974, p. 411ss).

35 Ibid., p. 412.
} 
En definitiva, el Derecho positivo tiene que tener en cuenta el punto de vista moral. Por eso, puede y debe impedir las consecuencias morales negativas. En este orden de cosas, Hildebrand señala que el papel de la esfera moral y de sus exigencias es para el derecho positivo más indirecto que el del Derecho natural. La ley moral exige que no se pase por encima de los derechos naturales a fin de evitar consecuencias moralmente malas. En caso de conflicto, el derecho positivo no puede pasar sin más por encima de los derechos de la persona a fin de evitar males morales. ${ }^{36}$ Esto pondría de manifiesto, según Hildebrand, precisamente desde el punto de vista moral, la prioridad de la consideración de los derechos naturales. El Estado no está moralmente justificado a ignorar los derechos naturales o a desproveer a los individuos de las facultades a las que tienen un derecho elemental.

Sin embargo, como el propio Reinach reconoce, aquí surgen también problemas de difícil solución. ¿Le estaría permitido al Derecho positivo en aras de una distribución justa anular el derecho de una persona a legar testamentariamente sus propiedades? ¿Podría el Estado conceder al necesitado un derecho a la propiedad de las riquezas porque es mejor que el rico dé a éste lo que le sobra? Estas cuestiones en las que, eventualmente, pueden entrar en conflicto la esfera de los derechos naturales con la de lo valioso o moralmente valioso no son nada fáciles. En cualquier caso, Hildebrand insiste en lo extremadamente cuidadoso que hay que ser en la delimitación del marco en el que el derecho positivo puede pasar por encima de los derechos naturales a causa de algo valioso. $^{37}$

Aquí hay que recordar de nuevo la necesidad de separar la esfera del derecho y la de la moral o valioso La esfera de la moral se extiende a esferas que escapan a la esfera del derecho. Es lo que sucede con el amor y con las obligaciones que éste funda. Sería absurdo transformar el deber de amar al prójimo en una obligación legal por medio de una ley positiva. En resumen y en palabras de Hildebrand:

\footnotetext{
36 Ibid., p. 413.

37 Ibid., pp. 413-414.
} 


\begin{abstract}
“La esfera legal tiene su propia ratio, y por mucho que el Derecho positivo tenga que tener en cuenta la cuestión acerca de los valores y, en particular, los bienes morales, el dominio del Derecho está delimitado y su norma tiene que ser, junto con reglas de utilidad, no primariamente la esfera de lo valioso y de lo moral, sino el Derecho natural, esta categoría de derechos (Rechtskategorie) con un toque moral pero que tiene su fundamento con independencia de los valores". 38
\end{abstract}

\title{
VI. Conclusiones
}

Son muchos los aspectos de la teoría del Derecho a priori, iniciada por Adolf Reinach y continuada por otros autores, entre ellos, Dietrich von Hildebrand, a los que no me he referido en este trabajo. No me he referido, por ejemplo, a la supuesta "falta de importancia" de esta teoría para el Derecho positivo objetada por Schambeck en tan sólo un par de líneas ${ }^{39} \mathrm{o}$ al "intuicionismo" reprochado por Poulantzas ${ }^{40}$ y Gardies. ${ }^{41}$ Como decía al inicio, mi objetivo era la reconstrucción sistemática y el análisis crítico del rendimiento de la crítica de la teoría del Derecho a priori al iusnaturalismo. Para ello es preciso tener en cuenta el carácter un tanto apresurado de esta crítica en la obra de Reinach. Su temprana muerte en el campo de batalla durante la Primera Guerra Mundial le impidió profundizar en ella. Por otra parte, no hay que ocultar que las nociones de "iusnaturalismo" y de "Derecho natural" que este autor maneja parecen restringirse a las teorías modernas del Derecho natural. Resulta, por ejemplo, dificil pensar que las objeciones realizadas por Reinach al uso del concepto de "naturaleza" o "natural" sean aplicables a Santo Tomás. Tampoco puedo entrar aquí en esta interesante cuestión. Lo que me interesaba poner de relieve es que las diferencias que Reinach ve entre su teoría del Derecho a priori y la teoría del Derecho natural llevan a plantear una cuestión de

\footnotetext{
38 Ibid., p. 414.

39 (Schambeck H. , 1964, p. 128).

40 (Poulantzas, 1963, p. 219).

41 (Gardies, 1972).
} 
innegable relevancia filosófica, a saber, la distinción y, al mismo tiempo, la relación entre las esferas de lo rechtlich y de lo sittlich.

El mérito de Reinach habría consistido en señalar una tercera vía frente al positivismo jurídico y al iusnaturalismo. Las entidades jurídicas descubiertas por Reinach poseen una validez independiente de que sean o no promulgadas por el poder legislativo y, al mismo tiempo, son puramente formales, esto es, son formas, estructuras de sentido. ${ }^{42}$ Dado que, como señala (Loidolt, 2010, p. 110), estas estructuras tienen que ser presupuestas para que las entidades jurídicas, también las del Derecho positivo, puedan ser comprendidas, la investigación de ellas que lleva a cabo Reinach tiene un carácter a priori, genético y trascendental (aunque esto último no fue pretendido directamente por él). Se trataría de una investigación trascendental porque estas estructuras que, por ejemplo, se encuentran en el prometer "no es tanto un a priori estático, sino un interaccionar intersubjetivo que funda relaciones" (Loidolt, 2010, p. 110). Pero ello ha de ser objeto de otro trabajo.

\footnotetext{
${ }^{42}$ Esto lo señala también (Reiner, 1969, p. 30) cuando caracteriza al método fenomenológico como una "tercera via" frente a aquellas teorías "metafísicas" que fundan el derecho y la moralidad en el presupuesto religioso-metafísico de la fe en Dios y en los mandamientos dados por Él o en la metafísica de una "estructura ontológica" o "esencia" del ser humano y frente a las teorías "anti-metafísicas", existiría una "tercera vía", a saber, la representada por el método fenomenológico.
} 


\section{REFERÊNCIAS}

Albert, M. (2013a). ¿Qué es el Derecho? La ontología jurídica de Adolf Reinach. Madrid: Centro de Estudios Políticos y Constitucionales.

- (2013b). Natural Law and the Phenomenological Given. En F. J. Contreras, The Threads of Natural Law. Unraveling a Philosophical Tradition, (pp. 107-121). Dordrecht: Springer.

Bobbio, N. (1958). 'La natura delle cose”. En N. Bobbio, Giusnaturalismo e positivismo giuridico.

Bodenheimer, E. (1969). "Phänomenologie und Rechtsquellenlehre". En T. Würtenberger, Phänomenologie, Rechtsphilosophie, Jurisprudenz. Festschrift für Gerhart Husserl zum 75 Geburtstag, (pp. 72-92). Frankfurt: Vittorio Lostermann.

- (2010). Einführung in die Rechtsphänomenologie. Eine historisch-systematische Darstellung. Tübingen: Mohr Siebeck.

Gardies, J. (1972). Essai sur les fondements a priori de la rationalité morale et juridique. Paris: Librairie Générale de Droit et de Jurisprudence.

Gutzwiller, M. (1965). "Zur Lehre von der "Natur der Sache”. En A. Kaufmann, Die ontologische Begründung des Rechts, (pp. 14-32). Darmstadt: Wissenschaftliche Buchgesellschaft.

Hildebrand, D. v. (1974). "Die rechtliche und sittliche Sphäre in ihrem Eigenwert und in ihrem Zusammenhang". En D. v. Hildebrand, Gesammelte Werke, Bd. VII, Idolkult und Gotteskult, (pp. 399-415). Regensburg: Verlag Josef Habbel.

Husserl, G. (1955). Recht und Zeit. Fünf rechtsphilosophische Essays. Frankfurt: Vittorio Klostermann.

Mayer-Maly, T. (1998). “Vergewisserung über Naturrecht”. En J. Seifert, Wie erkennt man Naturrecht?, (pp. 13-31). Heidelberg: Carl Winter Verlag.

Mitterer, A. (1931). "Naturwissenschaft und Naturrecht. Zusammenhänge bei Thomas von Aquin". En Die soziale Frage und der Katholizismus. Festschrift zum 40. Jubiläum der Enzyklika "Rerum novarum”, (p. 436ss). Paderborn.

Poulantzas, N. (1963). "Notes sur la phénoménologie et l'existentialisme juridiques". Archives de Philosophie du Droit (8).

Radbruch, G. (1990). "Die Natur der Sache als juristische Denkform”. En G. Radbruch, \& W. Hassemer (Ed.), Gesamtausgabe (Vol. III, pp. 229-254). Heidelberg: C.F. Müller.

Reinach, A. (1989). "Über Phänomenologie". En A. Reinach, \& B. S. Smith (Ed.), Sämtliche Werke. Textkritische Ausgabe in 2. Bänden, (pp. 531-550). München: Philosophia Verlag. 
- (1989). Die apriorischen Grundlagen des bürgerlichen Rechtes. En A. Reinach, \& K. S. B. Smith (Ed.), Sämtliche Werke. Textkritische Ausgabe in 2 Bänden. II, Kommentar und Textkritik (Vol. I, pp. 141-278). München: Philosophia Verlag.

- (1989). Einführung in die Philosophie. En A. Reinach, Sämtliche Werke.

Reinach, A. (2010). Los fundamentos a priori del Derecho civil. (M. Crespo, Trad.) Granada: Comares.

Reiner, H. (1969). "Zur Bedeutung der phänomenologischen Methode in Ethik und Rechtsphilosophie". En T. Würtenberg, Phänomenologie, Rechtsphilosophie, Jurisprudenz. Festschrift für Gerhart Husserl zum 75. Geburtstag, (pp. 27-37). Frankfurt: Vittorio Klostermann.

- (1964). Grundlagen, Grundsätze und Einzelnormes des Naturrechts. Freiburg: Karl Alber.

Schambeck, H. (1965). "Der Begriff der 'Natur der Sache”. En A. Kaufmann, Die ontologische Begründung des Rechts. Darmstadt: Wissenschaftliche Buchgesellschaft.

- (1964). Der Begriff der 'Natur der Sache'. Ein Beitrag zur rechtphilosophischen Grundlagenforschung. Wien: Springer Verlag.

Sowa, R. (2012). "Eidetics and its Methodology". En S. O. Luft, The Routledge Companion to Phenomenology, (pp. 254-265). Abingdon: Routledge.

Waldstein, W. (2010). Ins Herz geschrieben. Das Naturrecht als Fundament einer menschlichen Geselschaft. Augsburg: Sankt Ulrich Verlag.

- (1967). Vorpositive Ordnungselemente im römischen Recht. Antrittsvorlesung gehalten am 25. Jänner 1966 an der Universität Salzburg. (Vols. Salzburger Universitätsreden, Heft 19). Salzburg: Anton Pustet. 